\title{
Capsule Commentary on Kuczmarska et al., Detection of Delirium in Hospitalized Older General Medicine Patients: A Comparison of the 3S-CAM and CAM-ICU
}

\author{
Jennifer Fernandez, MD and Lee A. Lindquist, MD MPH MBA
}

Northwestern University Feinberg School of Medicine, Chicago, IL, USA.

J Gen Intern Med 31(3):324

DOI: $10.1007 / \mathrm{s} 11606-015-3546-5$

(c) Society of General Internal Medicine 2015

$\mathrm{D}$ etection of delirium is extremely important, but it is frequently missed. Indeed, one study reported that $76 \%$ of cases in the ED were missed. ${ }^{2}$ Therefore, having a brief assessment tool to ensure prompt recognition of delirium is critical. The CAM-ICU is a bedside assessment tool that is frequently used to detect delirium in non-verbal patients, although it has been validated for use in verbal patients as well. The 3D-CAM is a new assessment tool that can be completed in an average of $3 \mathrm{~min}$. This study by Kuczmarska et al. ${ }^{1}$ compared the diagnostic accuracy of the CAM-ICU to the 3D-CAM for detection of delirium in general medicine hospitalized patients. A reference standard was administered first to diagnose delirium, and the CAM-ICU and 3D-CAM were then randomly administered by research assistants, who were blinded to the results of the previous assessments. The authors found that the 3D-CAM had substantially higher sensitivity than the CAM-ICU (95\% vs. $53 \%$ ), whereas specificity for both was $>90 \%$. They also found that the 3DCAM and CAM-ICU required a similar length of time to administer.

In addition, subgroup analyses were performed in attempts to understand differences in sensitivity. Subgroup analysis of delirium severity found that the 3D-CAM had superior sensitivity in cases of mild delirium (100\% vs $30 \%$ ). The authors concluded that the CAM-ICU appeared to detect cases of more severe delirium, which is often the case in the ICU, whereas the 3D-CAM may be better for patients on the floor. Recent studies have shown lower sensitivity in verbal patients with CAM-ICU use. ${ }^{2,3}$ A study by Hans et al. showed sensitivity of $68-72 \%$ in ED patients. ${ }^{2}$ Further work is needed to examine the use of the CAM-ICU in non-ICU populations.

The authors of this study concluded that the 3D-CAM may be a superior screening tool, especially for detecting milder cases of delirium in general medicine patients. This is clinically significant, as cases of delirium, especially mild or hypoactive delirium, are frequently missed or underdiagnosed, and having a fast screening tool will likely lead to increased diagnosis of delirium.

Corresponding Author: Jennifer Fernandez, MD; Northwestern University Feinberg School of Medicine, Chicago, IL 60611, USA (e-mail: jennifer.fernandez@northwestern.edu).

\section{Compliance of Ethical Standards:}

Conflicts of Interest: The authors have no conflict of interest with this article.

\section{REFERENCES}

1. Kuczmarska A, Ngo LH, Guess J, O'Connor MA, Branford-White L, Palihnich K, Gallagher $\mathbf{J}$, Marcantonio E. Detection of delirium in hospitalized older general medicine patients: a comparison of the 3S-CAM and CAM-ICU. J Gen Intern Med. 2015. doi:10.1007/s11606-015-3514-0.

2. Han JH, WA, Graves AJ, Shintani A, Schnelle JF, Dittus RS, et al. Validation of the Confusion Assessment Method for the Intensive Care Unit in older emergency department patients. Acad Emerg Med. 2014; 21: p. 180-7.

3. Neufeld KJ, et al. Evaluation of two intensive care delirium screening tools for non-critically ill hospitalized patients. Psychosomatics. 2011;52(2):133-40.

Published online December 2, 2015 